\title{
SUDDEN VISUAL LOSS IN ACUTE LEUKAEMIA
}

\author{
Tengku Kamalden' TA, Nurliza $K^{\prime}$, Haris $A R^{2}$ \\ I Department of Ophthalmology, University of Malaya Medical Centre, 50603 Kuala Lumpur, Malaysia \\ 2 Haematology Unit, Department of Medicine, University of Malaya Medical Centre, 50603 Kuala Lumpur, Malaysia
}

\begin{abstract}
The presenting signs of acute leukaemia occur as a result of bone marrow failure and organ infiltration. Increased bleeding tendencies are seen on the skin, gums and mucosal lining. Bleeding in the posterior segment of the eye, namely the retina and vitreous, may occur, but do not usually cause any visual disturbances. This case demonstrates visual loss as a result of premacular subhyaloid haemorrhage in acute leukaemia. (JUMMEC 2008; 11(1): 30-32)
\end{abstract}

KEYWORDS: Sudden visual loss, subhyaloid haemorrhage, acute leukaemia

\section{Case Report}

An Nd:YAG posterior hyaloidotomy was given on two separate occasions to the right submacular haemorrrhage, but failed to drain the blood effectively into the vitreous cavity. Despite this, her right vision improved to I/60. She was commenced on high-risk UK ALL protocol regime with minimal other complications, and achieved complete remission a month later. The retinal haemorrhages in the left eye were absorbed within six weeks, while the right dense subhyaloid haemorrhage took about four months to completely resolve. Her best corrected vision on right eye steadily improved to $6 / 9$, while vision on her left eye was never affected.

\section{Discussion}

Subhyaloid haemorrhage is defined by its location between the vitreous and the retina. Separation of vitreous from the retina by a collection of blood in the macula region, otherwise known as premacular subhyaloid haemorrhage, may cause sudden loss of vision. Aetiologies include proliferative diabetic retinopathy, retinal venous occlusion, rupture of retinal macroaneurysms, valsalva retinopathy and hematological malignancies. Rennie and colleagues reported that resolution of premacular subhyaloid haemorrhage may occur from 3 to 18 months, depending on the aetiology $(I)$. In this case, submacular haemorrhage most likely occurred following bouts of non-productive cough in this patient with bleeding tendencies secondary to thrombocytopenia. Reddy and Jackson reported a significant association between intraretinal haemorrhages and thrombocytopenia in patients with acute lymphablastic leukaemia (ALL) (2). In their study, approximately half of the patients with ALL have retinal manifestations, of which intraretinal haemorrhages and white-centred haemorrhages were the most common. In their earlier study, Reddy and colleagues had demonstrated that the presence of intraretinal haemorrhage was a poor prognostic factor for survival in acute leukaemia (3).

Haemorrhages occurring in the macula lead to visual disturbance, and may cause visual loss if the size of bleed is large. Different techniques have been described to treat premacular haemorrhages. Among these are pars plana vitrectomy (4), intravitreal injection of gas and tissue plasminogen activator (5), and laser posterior hyaloidotomy. Puncturing the posterior hyaloid face with Nd:YAG provides a safe, yet non-invasive and effective treatment (6). This method was first described by Faulborn in 1988 for an eye with diabetic retinopathy (7). Nili-Ahmadabadi and colleagues reported a $100 \%$ success rate with this method, with best improvement noted in patients with Valsalva retinopathy $(6,8)$. Complications are uncommon, and include macular hole and retinal detachment (8).

Correspondence:

Nurliza K.

Department of Ophthalmology

Faculty of Medicine

University of Malaya

50603 Kuala Lumpur, Malaysia 


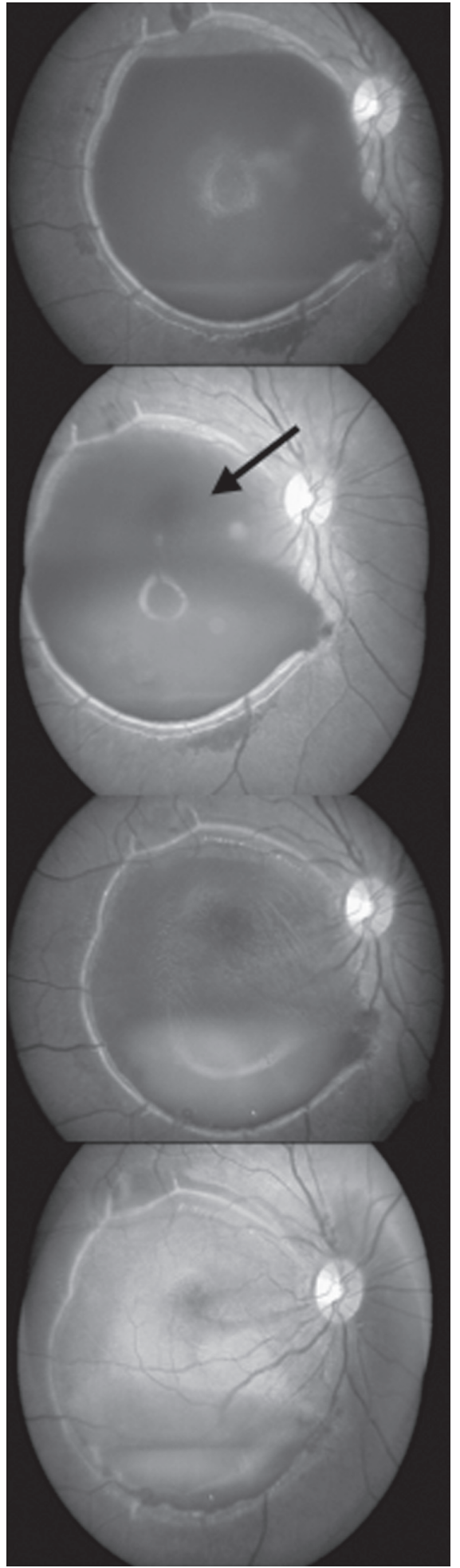

A

B

$\mathrm{C}$

$\mathrm{D}$

Figure I. Serial photographs of the right fundus. A. A fresh premacular subhyaloid haemorrhage on presentation with visual acuity of couting fingers. A denser level of blood clot was seen as the inferior aspect. B. Partial clearing of the haemorrhage with visualization of the macula (arrow) immediately after posterior hyaloidotomy with $\mathrm{Nd}$ :YAG laser performed four days later. C. Slow resolution with formation of blood clot two months later. D. There was almost complete absorption of the blood clot three months after presentation.

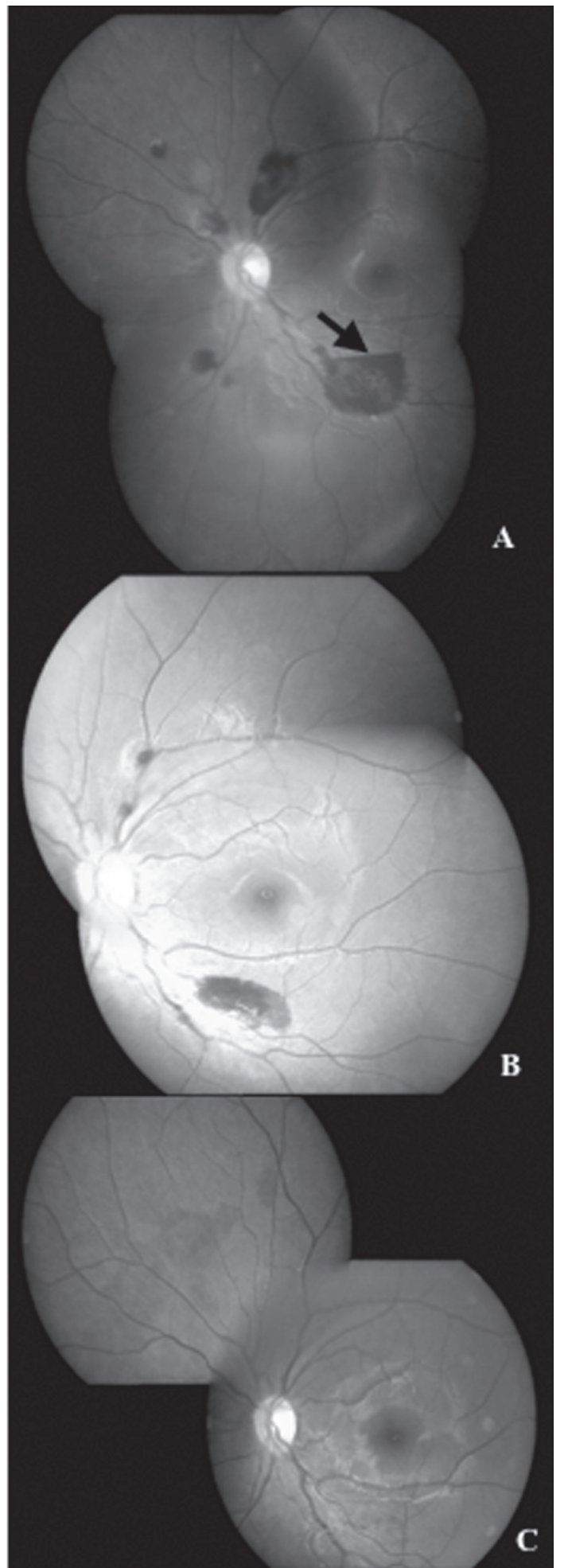

Figure 2. Serial photographs of the left fundus. A. On presentation, there were multiple intraretinal haemeorrhages with a small area of subhyloaid haemorrhage (arrow) inferotemporal to the macula. Fovea was spared and visual acuity was $6 / 6$. B. Two weeks later, the subhyaloid haemorrhage was absorbed leaving residual retinal haemorrhage. C. Complete resolution of retinal haemorrhages occurs about six weeks after presentation. 
In summary, this case illustrates visual loss as part of the presenting symptoms of acute leukaemia, caused by premacular subhyaloid haemorrhage. Laser posterior hyaloidotomy provides a good and safe alternative mode of treatment.

\section{Acknowledgements}

The authors would like to thank Cik Faizah Othman from the Ophthalmology Clinic, University of Malaya Medical Centre, for providing the fundus photographs.

\section{References}

I. Rennie CA, Newman DK, Snead MP, et al. Nd-YAG laser treatment for premacular submacular haemorrhage. Eye 2001; 15:519-524.

2. Reddy SC, Jackson N. Retinopathy in acute leukaemia at initial diagnosis: correlation of fundus lesions and haematological parameters. Acta Ophthalmol Scand 2004; 82:8I-85.
3. Reddy SC, Quah SH, Low HC, et al. Prognostic significance of retinopaty at presentation in adult acute leukaemia. Ann Hematol 1998; 76:15-18.

4. O'Hanley GP, Canny CL. Diabetic dense premacular hemorrhage. A possible indication of prompt vitrectomy. Ophthalmology 1985; 92:507-5I I.

5. Conway MD, Peyman GA, Recasens M. Intravitreal tPA and SF6 promote clearing of premacular subhyaloid hemorrhages in shaken and battered baby syndrome. Ophthalmic Surg Lasers 1999; 30:435-44I.

6. Nili-Ahmadabadi M, Lashay A-R, Karkhaneh R, et al. Nd-YAG laser application in premacular subhyalod hemorrhage. Arch Iranian Med 2004; 7(3):206-209.

7. Faulborn J. Behandlung einer diabetischen premaculaeren Blutung mit dem Q-switched Neodym: YAG laser. Spektrum Augenheilkd 1988; 2:33-35.

8. Ulbig MW, Mangouritsas G, Hermann-Rothbacher $\mathrm{H}$, et al. Long-term results after drainage of premacular subhyaloid hemorrhage into the vitreous with a pulsed Nd:YAG laser. Arch Ophthalmol 1998; I | 6: | 465-469. 